\title{
GEOPHYTES OF IĞDIR (EAST ANATOLIA) AND THEIR ECONOMIC POTENTIALITIES AS ORNAMENTAL PLANT
}

\author{
Ernaz Altundağ Çakır ${ }^{1 *}$ \\ 1*Department of Biology Faculty of Arts and Science, Duzce University, 81620 Konuralp, Duzce. E-mail: \\ ernazaltundag@duzce.edu.tr
}

\begin{abstract}
Geophytes are the most preferred group among the ornamental plants due to their aesthetic features, suitability to be cut flowers and their fragrance. The aim of the research is to identify geophyte taxa, their risk categories and economic potentialities as ornamental plants in Iğdır province. Plant specimens were collected from Iğdır province between 2007 and 2012 vegetation seasons. The collected plant specimens were kept in ISTE (the herbarium of the Istanbul University, Faculty of Pharmacy). As a result of this study, 52 geophytic taxa belonging to 12 families are determined from the investigation area. 4 taxa are endemic (Allium armenum, A. baytopiorum, Bellevalia gracilis and Pseudomuscari forniculatum) to Turkey. According to floristic regions, 32 Irano-Turanian elements are ranked first, followed by 6 Mediterranean elements and 4 Euro-Siberian elements. 10 of the identified species are widespread or unknown phytogeographic origin. The most richest families are Asparagaceae (14 taxa), Amaryllidaceae ( 9 taxa), Liliaceae ( 9 taxa), Iridaceae (5 taxa), Asteraceae (3 taxa), Orchidaceae (3 taxa). Of all the collected taxa, 61\% were bulbous, $17 \%$ were rhizomatous, $10 \%$ were tuberous, $8 \%$ were tuberous roots and $4 \%$ were cormous. Owing to the attractive flowers, 43 taxa were signed as ornamental plant potentialities.
\end{abstract}

Keywords: Geophyte, ornamental plant, economic plant, Iğdır, East Anatolia, Turkey

\section{Özet}

Geofitler estetik özellikleri, kesme çiçekçiliğe uygun olmaları ve hoş kokuları sebebiyle süs bitkisi olarak en fazla tercih edilen bitkilerdir. Bu çalışma Iğdır ilinin geofitleri ve bunların süs bitkisi olarak ekonomik açıdan değerlendirilebilme potansiyelleri üzerine hazırlanmıştır. Bitki örnekleri 2007-2012 yılları arasında farklı vejetasyon dönemlerinde toplanmıştır. Toplanan bitki örnekleri ISTE'de (İstanbul Üniversitesi Eczacılık Fakültesi Herbaryumu) saklanmaktadır. Çalışma sonucunda 12 familyaya ait 52 geofit taksonu saptanmıştır. Bunlardan 4 tanesi endemiktir (Allium armenum, A. baytopiorum, Bellevalia gracilis and Pseudomuscari forniculatum). Taksonlar fitocoğrafik bölgelere göre gruplandırıldığında 32 takson Iran-Turan elementi, 6 takson Akdeniz elementi, 4 takson Avrupa-Sibirya elementi ve 10 taksonun ise fitocoğrafik bölgesi bilinmemektedir. En fazla taskonla temsil edilen familyalar sirasiyla; Asparagaceae (14 takson), Amaryllidaceae (9 takson), Liliaceae (9 takson), Iridaceae (5 takson), Asteraceae (3 takson), Orchidaceae (3 takson)'dir. Geofit tiplerine göre taksonların \%61'i soğanl1, \%17'si rizomlu, \%10’u tuberli, \%8'i tuber köklü ve \%4 tanesi de kormludur.

Anahtar Sözcükler: Geofit, süs bitkisi, ekonomik bitki, Iğdır, Doğu Anadolu, Türkiye.

\section{INTRODUCTION}

Geophytes are plants that survive part of their annual life cycle as a dormant, fleshy underground structure. Types of geophytes include bulbs, corms, tubers, tuberous stems, tuberous roots, rhizomes and pseudobulbs (Kamenetsky and Hiroshi 2013). There are 8796 species in Flora of Turkey (excluding an additional 192 species of The East Aegean Islands) (Davis 1965-1985, Davis et al. 1988, Güner et al. 2000). According to the last checklist, an additional 945 species were added flora of Turkey (Özhatay et al. 2013). The rate of endemism is about \%34 in the flora of Turkey (Davis 1965). Geophytes form a significant part of the biological richness for our country. The geophytes are represented by approximately 600 
species, with almost $40 \%$ of them are endemic (Davis 1965-1985).

The study area, Iğdır province is located in the Erzurum-Kars part of the Eastern Anatolian region of Turkey. Its area covers $3539 \mathrm{~km}^{2}$ and the elevation of its land varies from 795 to 5165 $\mathrm{m}$. Iğdır has $74 \%$ of the mountains and high plateaus and $26 \%$ of lowlands and has remarkable diversity about geophytes because of the mountains and high plateaus (Altundağ 2012).

Most of geophytes of Turkey prefer high altitudes and wetlands for wide spreading. Mount Ararat (5165 m), Zor (3196 m), Kizilcaziyaret $(2887 \mathrm{~m})$, Durak $(2811 \mathrm{~m})$ and Tekalt1 $(2560 \mathrm{~m})$ mountains and some of the wetlands in Aras valley have great diversity for geophytes in the province (Altundağ 2012).

Geophytes have economic value due to their attractive flowers and usage in the drug industry (Ekim et al. 1991). Geophytes are the most preferred group among the ornamental plants due to their aesthetic features, suitability to be cut flowers and their fragrance (Ç. $\breve{g}$ and Başdoğan 2015). There is some research about the geophytes in different areas of Turkey (Çelik et al. 2004, Eker et al. 2008, Özuslu and İskender 2009, Çingay et al. 2012, Sargin et al. 2013, Korkmaz et al. 2014, Firat et al. 2015, Avcu et al. 2016 and Demirelma and Ertuğrul 2016,) but no investigation about Iğdır Province. This study aims to describe geophytic flora and their economic potentialities as ornamental plants in Iğdır province.

\section{MATERIAL AND METHODS}

Plant specimens were collected from Iğdır province between 2007 and 2012 vegetation seasons. The collected plant specimens were kept in ISTE (the herbarium of the Istanbul University, Faculty of Pharmacy). These specimens were identified basically with the Flora of Turkey (Davis et al. 1984, Davis et al. 1988, Güner et al. 2000). Furthermore, TÜBIVES (www.tubives.com) was scanned for missing taxa of Iğdır province. Scientific plant names were checked by using Plant List website (www.theplantlist.org). The complete geophyte list was given in Table 1. In the table, the following details are provided as scientific and family name, herbarium number or voucher specimen; type of geophyte; endemism and threatened category, phytogeographical region, and plants which have attractive flowers were signed for their economic potentialities as ornamental plants. Endemic and rare taxa were categorized according to new Red Data categories (Ekim et al. 2000).

\section{RESULTS AND DISCUSSION}

As a result of this study, fifty two geophytic taxa belonging to twelve families and twenty seven genera are determined from the investigation area. Allium armenum, A. baytopiorum, Bellevalia gracilis and Pseudomuscari forniculatum (Figure 1) are endemic to Turkey. According to floristic regions, thirty two Irano-Turanian elements are ranked first, followed by six Mediterranean elements and four Euro-Siberian elements. The large number of Irano-Turanian elements can be explained by the fact that the study area lies completely within the Irano-Turanian phytogeographical region. Ten of the identified species are widespread or unknown phytogeographic origin.

In addition, forty six of the collected taxa are determined to be Liliopsida and the remaining six are Magnoliopsida. The most richest families are Asparagaceae (14 taxa), Amaryllidaceae (9 taxa), Liliaceae (9 taxa), Iridaceae (5 taxa), Asteraceae (3 taxa), Orchidaceae (3 taxa) (Figure $2)$. In terms of taxa number, the major genera in the province are as follows: Allium (9 taxa), Gagea (5 taxa), Bellevalia (3 taxa), Iris (3 taxa), Ornithogalum (3 taxa) (Figure 3). Of all the collected taxa, 61\% were bulbous, $17 \%$ were rhizomatous, $10 \%$ were tuberous, $8 \%$ were tuberous roots and $4 \%$ were cormous (Figure 4 ). Owing to the attractive flowers, forty three taxa (Allium atroviolaceum, A. armenum, A. cardiostemon, A. subakaka, Anacamptis palustris, Asparagus palaestinus, A. persicus, Asphodeline prolifera, Bellevalia gracilis, B. paradoxa, B. speciosa, Colchicum szovitsii, Corydalis erdelii (Figure 5), Dactylorbiza romana subsp. georgica, D. umbrosa, Eremurus spectabilis, Ficaria fascicularis, Fritillaria caucasica, F. pinardii, Gagea bohemica, G. bulbifera, G. glacialis, G. luteoides, G. reticulata, Geranium tuberosum, Gladiolus atroviolaceus G. kotschyanus, Iris caucasica (Figure 6), I. iberica subsp. elegantissima, I. spuria L. subsp. musulmanica, Leopoldia comosa, Merendera trigyna, Muscari armeniacum, $M$. caucasicum, Ornithogalum montanum, $O$. narbonense, $O$. oligophyllum, Pseudomuscari forniculatum, Pulsatilla armena, Puschkinia scilloides (Figure 7), Scilla siberica subsp. armena (Figure 8), Tulipa armena and T. biflora) were signed as ornamental plant potentialities (Table 1). 
Table 1. List of geophytes in Iğdır province

\begin{tabular}{|c|c|c|c|c|}
\hline $\begin{array}{l}\text { Scientific \&Family names; } \\
\text { Herbarium number (ISTE) or } \\
\text { Voucher specimen }\end{array}$ & $\begin{array}{l}\text { Type of } \\
\text { Geophyte }\end{array}$ & $\begin{array}{l}\text { Endemism/T } \\
\text { hreatened } \\
\text { category }\end{array}$ & $\begin{array}{l}\text { Phytogeog. } \\
\text { Region }\end{array}$ & $\begin{array}{l}\text { Economic } \\
\text { potential as } \\
\text { ornamenta } \\
\text { plant }\end{array}$ \\
\hline $\begin{array}{l}\text { Allium armenum Boiss. \& Kotschy } \\
\text { Amaryllidaceae } 85600\end{array}$ & Bulb & $\mathrm{EN} / \mathrm{LC}$ & Ir.-Tur. & + \\
\hline $\begin{array}{l}\text { Allium atroviolaceum Boiss. } \\
\text { Amaryllidaceae } 85867\end{array}$ & Bulb & $-/-$ & - & + \\
\hline $\begin{array}{l}\text { Allium baytopiorum Kollmann \& } \\
\text { Özhatay Amaryllidaceae TÜBİVES }\end{array}$ & Bulb & $\mathrm{EN} / \mathrm{EN}$ & Ir.-Tur. & - \\
\hline $\begin{array}{l}\text { Allium cardiostemon Fisch. \& } \\
\text { C.A.Mey. Amaryllidaceae } 85530\end{array}$ & Bulb & $-/-$ & Ir.-Tur. & + \\
\hline $\begin{array}{l}\text { Allium dictyoprasum C.A.Mey. ex } \\
\text { Kunth Amaryllidaceae TÜBİVES }\end{array}$ & Bulb & $-/-$ & Ir.-Tur. & - \\
\hline $\begin{array}{l}\text { Allium flavum L. Amaryllidaceae } \\
85733\end{array}$ & Bulb & $-/-$ & Medit. & - \\
\hline $\begin{array}{l}\text { Allium pseudoflavum Vved. } \\
\text { Amaryllidaceae TÜBİVES }\end{array}$ & Bulb & $-/-$ & Ir.-Tur. & - \\
\hline Allium scorodoprasum L. 84421 & Bulb & $-/-$ & Medit. & - \\
\hline $\begin{array}{l}\text { Allium subakaka Razyfard \& Zarre } \\
\text { Amaryllidaceae } 84598\end{array}$ & Bulb & $-/-$ & Ir.-Tur. & + \\
\hline $\begin{array}{l}\text { Anacamptis palustris (Jacq.) } \\
\text { R.M.Bateman, } \\
\text { Pridgeon\&M.W.Chase Orchidaceae } \\
\text { TÜBIVES }\end{array}$ & Tuber & $-/-$ & - & + \\
\hline $\begin{array}{l}\text { Asparagus palaestinus Baker } \\
\text { Asparagaceae TÜBİVES }\end{array}$ & Rhizome & $-/-$ & Medit. & + \\
\hline $\begin{array}{l}\text { Asparagus persicus Baker Asparagaceae } \\
85271\end{array}$ & Rhizome & $-/-$ & Ir.-Tur. & + \\
\hline $\begin{array}{l}\text { Asphodeline prolifera (M. Bieb.) Kunth } \\
\text { Xanthorrhoeaceae TÜBİVES }\end{array}$ & Rhizome & $-/-$ & Ir.-Tur. & + \\
\hline $\begin{array}{l}\text { Bellevalia gracilis Feinbrun } \\
\text { Asparagaceae } 85314\end{array}$ & Bulb & $\mathrm{EN} / \mathrm{LC}$ & Ir.-Tur. & + \\
\hline $\begin{array}{l}\text { Bellevalia paradoxa (Fisch.\&C.A.Mey.) } \\
\text { Boiss. Asparagaceae } 84409\end{array}$ & Bulb & $-/-$ & Ir.-Tur. & + \\
\hline $\begin{array}{l}\text { Bellevalia speciosa Woronow ex } \\
\text { Grossh. Asparagaceae } 84404\end{array}$ & Bulb & $-/-$ & - & + \\
\hline $\begin{array}{l}\text { Cirsium rhizocephalum C.A. Mey. } \\
\text { Asteraceae } 85447\end{array}$ & & $-/-$ & Ir.-Tur. & - \\
\hline $\begin{array}{l}\text { Colchicum szovitsii Fisch.\&C.A.Mey. } \\
\text { Colchicaceae } 85328\end{array}$ & Bulb & $-/-$ & Ir.-Tur. & + \\
\hline $\begin{array}{l}\text { Corydalis erdelii Zucc. Papaveraceae } \\
85327\end{array}$ & Tuber & $-/-$ & - & + \\
\hline $\begin{array}{l}\text { Dactylorbiza romana subsp. georgica } \\
\text { (Klinge) Soo ex Renz \& } \\
\text { Taubenheim Orchidaceae TÜBIVES }\end{array}$ & Tuber & $-/-$ & Euro-Sib. & + \\
\hline $\begin{array}{l}\text { Dactylorbiza umbrosa (Kar.\&Kir.) } \\
\text { Nevski Orchidaceae } \\
84420\end{array}$ & Tuber & $-/-$ & Ir.-Tur. & + \\
\hline $\begin{array}{l}\text { Eremurus spectabilis M.Bieb. } \\
\text { Xanthorrhoeaceae } 84401\end{array}$ & Rhizome & $-/-$ & Ir.-Tur. & + \\
\hline $\begin{array}{l}\text { Ficaria fascicularis K.Koch } \\
\text { Ranunculaceae }\end{array}$ & $\begin{array}{l}\text { Tuberous } \\
\text { root }\end{array}$ & $-/-$ & Ir.-Tur. & + \\
\hline
\end{tabular}




\begin{tabular}{|c|c|c|c|c|}
\hline $\begin{array}{l}\text { Fritillaria caucasica Adam Liliaceae } \\
\text { TÜBIVES }\end{array}$ & Bulb & $-/-$ & Euro-Sib. & + \\
\hline $\begin{array}{l}\text { Fritillaria pinardii Boiss. Liliaceae } \\
\text { TÜBİVES }\end{array}$ & Bulb & $-/-$ & Ir.-Tur. & + \\
\hline $\begin{array}{l}\text { Gagea bohemica (Zauschn.) } \\
\text { Schult.\&Schult.f. Liliaceae } 84411\end{array}$ & Bulb & $-1-$ & - & + \\
\hline $\begin{array}{l}\text { Gagea bulbifera (Pall.) Salisb. Liliaceae } \\
\text { TÜBİVES }\end{array}$ & Bulb & $-1-$ & Euro-Sib. & + \\
\hline $\begin{array}{l}\text { Gagea glacialis K.Koch Liliaceae } \\
84413\end{array}$ & Bulb & $-/-$ & Ir.-Tur. & + \\
\hline $\begin{array}{l}\text { Gagea luteoides Stapf Liliaceae } \\
\text { TÜBIVES }\end{array}$ & Bulb & $-/-$ & - & + \\
\hline $\begin{array}{l}\text { Gagea reticulata (Pall.) } \\
\text { Schult.\&Schult.f. Liliaceae } \\
85280\end{array}$ & Bulb & $-/-$ & Ir.-Tur. & + \\
\hline $\begin{array}{l}\text { Geranium tuberosum L. Geraniaceae } \\
85317\end{array}$ & Tuber & $-/-$ & - & + \\
\hline $\begin{array}{l}\text { Gladiolus atroviolaceus Boiss. Iridaceae } \\
85588\end{array}$ & Corm & $-/-$ & Ir.-Tur. & + \\
\hline $\begin{array}{l}\text { Gladiolus kotschyanus Boiss. Iridaceae } \\
84406\end{array}$ & Corm & $-1-$ & Ir.-Tur. & + \\
\hline Iris cancasica Hoffm. Iridaceae 85313 & Rhizome & $-/ \mathrm{VU}$ & Euro-Sib. & + \\
\hline $\begin{array}{l}\text { Iris iberica subsp. elegantissima (Sosn.) } \\
\text { Fed.\&Takht. Iridaceae } \\
85276\end{array}$ & Rhizome & $-/ \mathrm{VU}$ & Ir.-Tur. & + \\
\hline $\begin{array}{l}\text { Iris spuria L. subsp. musulmanica } \\
\text { (Fomin) Takht. Iridaceae } \\
85498\end{array}$ & Rhizome & $-1-$ & Ir.-Tur. & + \\
\hline $\begin{array}{l}\text { Leopoldia comosa (L.) Parl. } \\
\text { Asparagaceae } 84414\end{array}$ & Bulb & $-1-$ & Medit. & + \\
\hline $\begin{array}{l}\text { Merendera trigyna Woronow } \\
\text { Colchicaceae TÜBİVES }\end{array}$ & Bulb & $-/-$ & Ir.-Tur. & + \\
\hline $\begin{array}{l}\text { Muscari armeniacum Leichtlin ex Baker } \\
\text { Asparagaceae } 84403\end{array}$ & Bulb & $-/-$ & - & + \\
\hline $\begin{array}{l}\text { Muscari caucasicum (Griseb.) Baker } \\
\text { Asparagaceae } 85304\end{array}$ & Bulb & $-1-$ & Ir.-Tur. & + \\
\hline $\begin{array}{l}\text { Ornithogalum montanum Cirillo } \\
\text { Asparagaceae } 85559\end{array}$ & Bulb & $-/-$ & Medit. & + \\
\hline $\begin{array}{l}\text { Ornithogalum narbonense } \mathrm{L} . \\
\text { Asparagaceae } 84415\end{array}$ & Bulb & $-/-$ & Medit. & + \\
\hline $\begin{array}{l}\text { Ornithogalum oligophyllum E.D.Clarke } \\
\text { Asparagaceae } 84408\end{array}$ & Bulb & $-/-$ & - & + \\
\hline $\begin{array}{l}\text { Pseudomuscari forniculatum (Fomin) } \\
\text { Garbari Asparagaceae } 84405\end{array}$ & Bulb & $\mathrm{EN} / \mathrm{LC}$ & Ir.-Tur. & + \\
\hline $\begin{array}{l}\text { Pulsatilla armena } \text { Rupr. Ranunculacae } \\
84475\end{array}$ & Rhizome & $-1-$ & Ir.-Tur. & + \\
\hline $\begin{array}{l}\text { Puschkinia scilloides Adams } \\
\text { Asparagaceae } 84417\end{array}$ & Bulb & $-/-$ & Ir.-Tur. & + \\
\hline $\begin{array}{l}\text { Scilla siberica subsp. armena (Grossh.) } \\
\text { Mordak Asparagaceae } 84600\end{array}$ & Bulb & $-/-$ & Ir.-Tur. & + \\
\hline $\begin{array}{l}\text { Scorzonera mollis Bieb. subsp. sqowitzii } \\
\text { (DC.) Chamberlain Asteraceae } \\
84489\end{array}$ & $\begin{array}{l}\text { Tuberous } \\
\text { root }\end{array}$ & $-/-$ & Ir.-Tur. & - \\
\hline $\begin{array}{l}\text { Scorzonera suberosa K.Koch } \\
\text { Asteraceae } 84502\end{array}$ & $\begin{array}{l}\text { Tuberous } \\
\text { root }\end{array}$ & $-/-$ & Ir.-Tur. & - \\
\hline
\end{tabular}




\begin{tabular}{|l|l|l|l|l|}
\hline $\begin{array}{l}\text { Triglochin maritima L. Juncaginaceae } \\
85564\end{array}$ & Rhizome & $-/-$ & - & - \\
\hline Tulipa armena L. Liliaceae 84416 & Bulb & $-/-$ & Ir.-Tur. & + \\
\hline Tulipa biflora Pall. Liliaceae 85311 & Bulb & - -VU & Ir.-Tur. & + \\
\hline
\end{tabular}

Phytogeog.: Phytogeographical; EN: Endemic; LC: Least concern; VU: Vulnerable; Ir.-Tur.: Irano-

Turanian; Medit.: Mediterranean; Euro-Sib.: Euro-Siberian

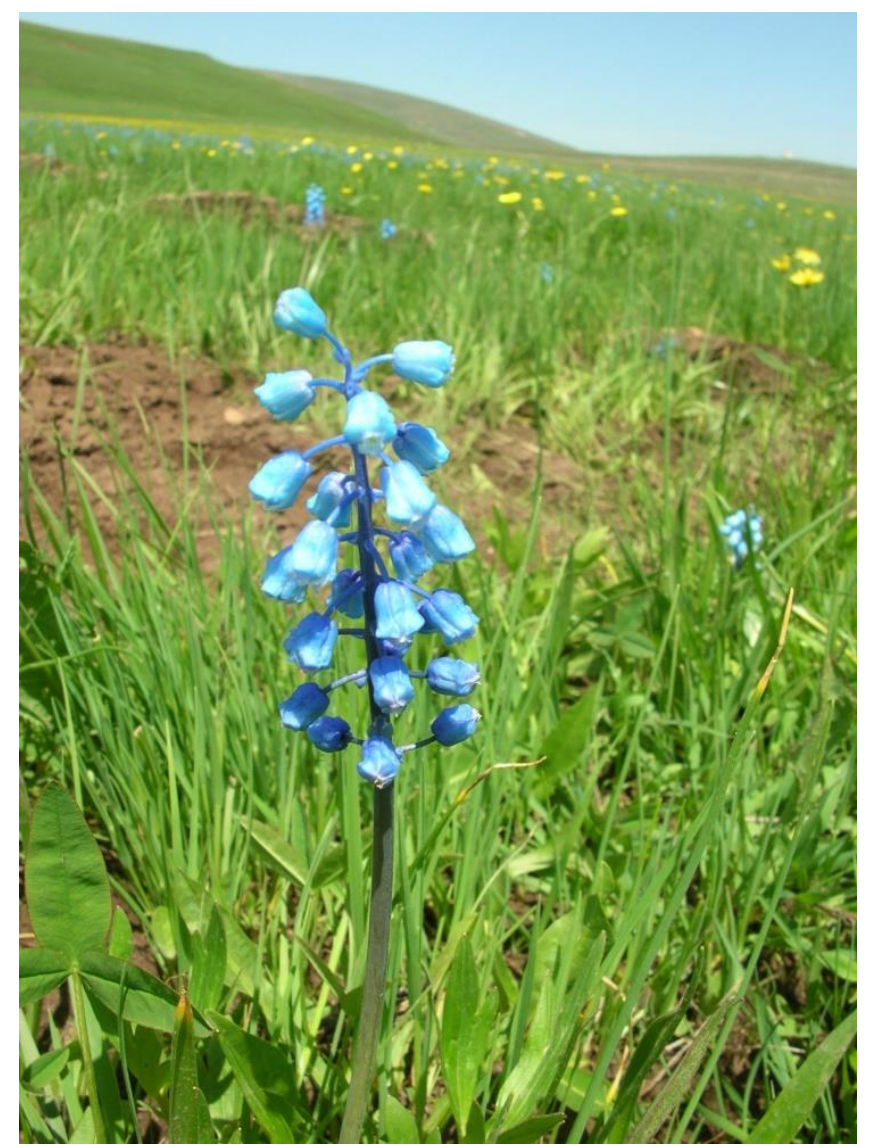

Figure 1. Pseudomuscari forniculatum, $2158 \mathrm{~m}$

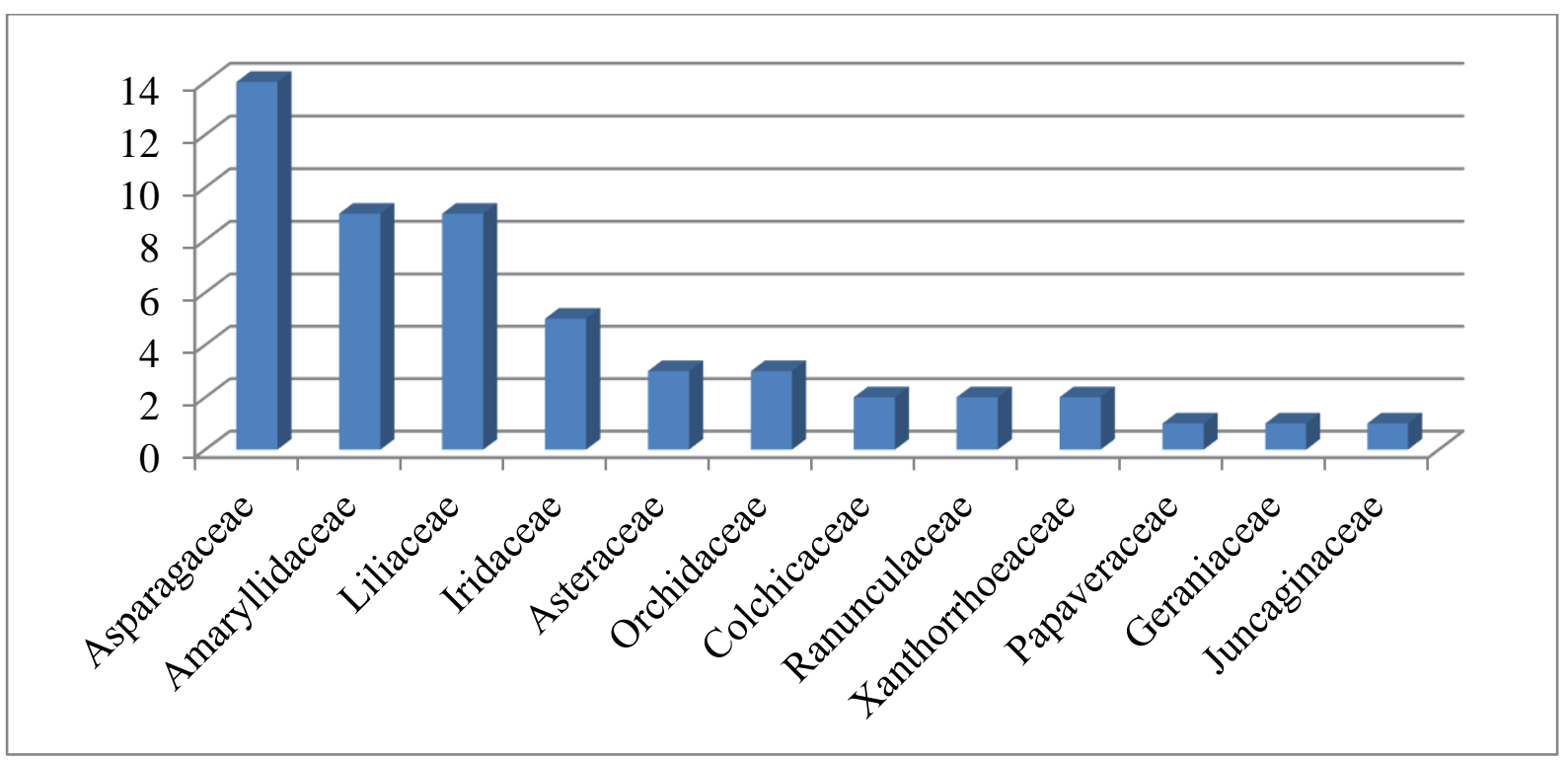

Figure 2. The most richest families 


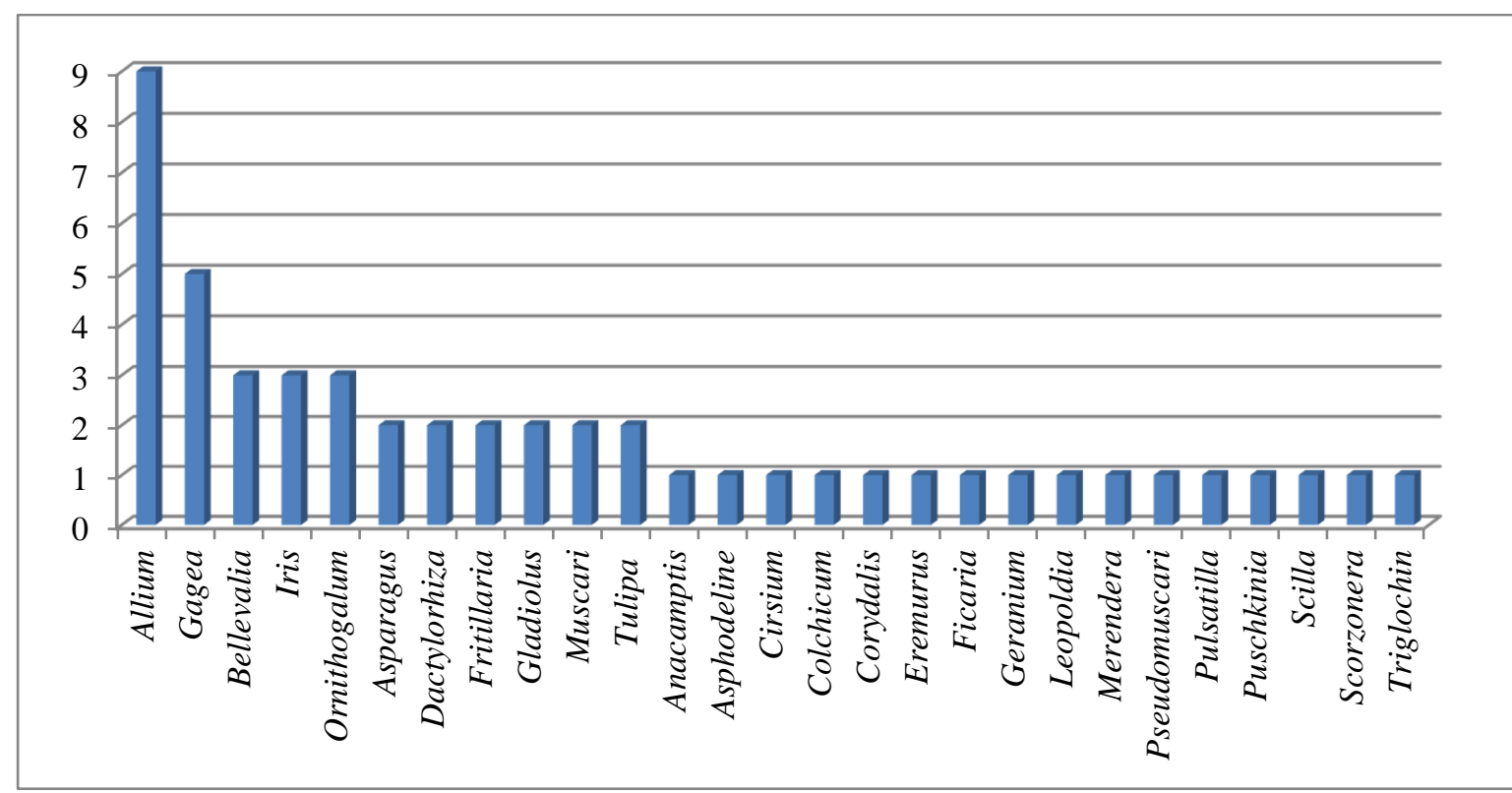

Figure 3. The most richest genera

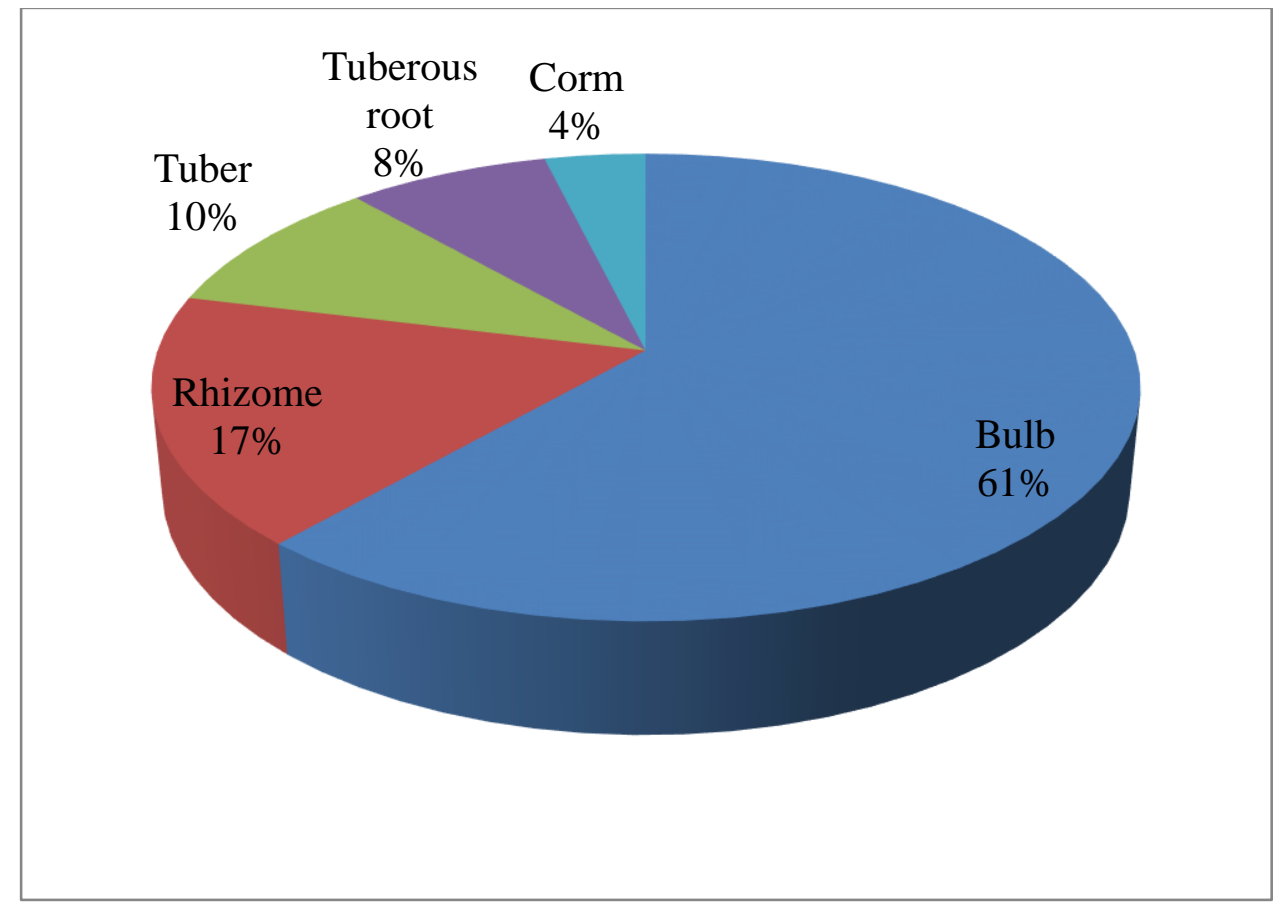

Figure 4. Geophyte types 


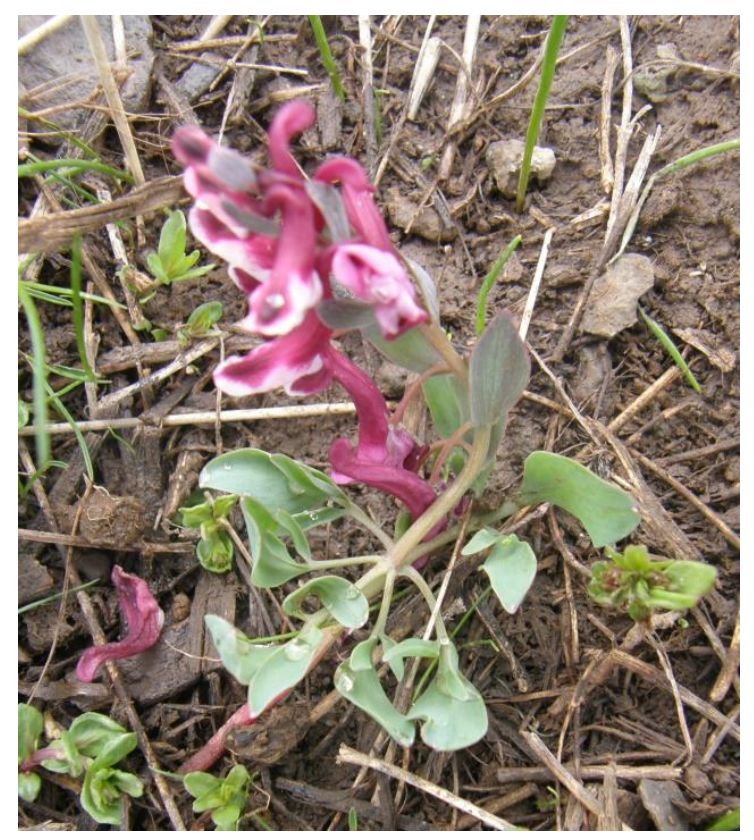

Figure 5. Corydalis erdelii, $2385 \mathrm{~m}$

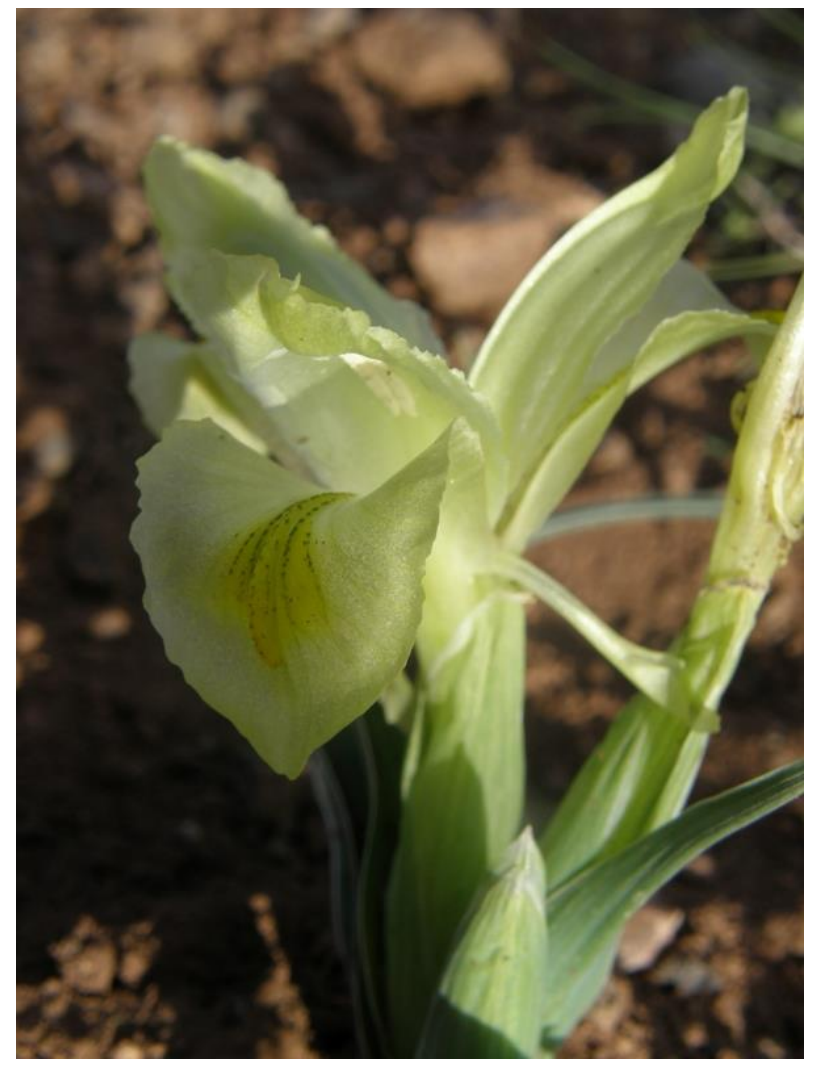

Figure 6. Iris cancasica, $2216 \mathrm{~m}$ 


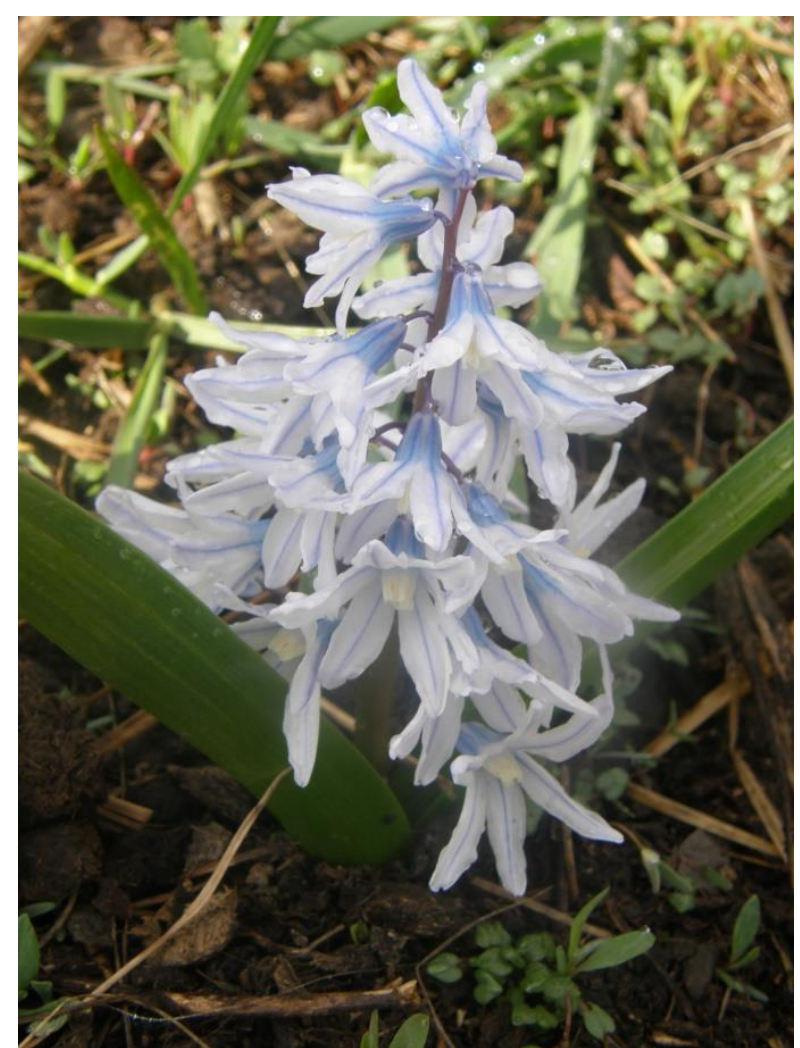

Figure 7. Puschkinia scilloides, $2252 \mathrm{~m}$

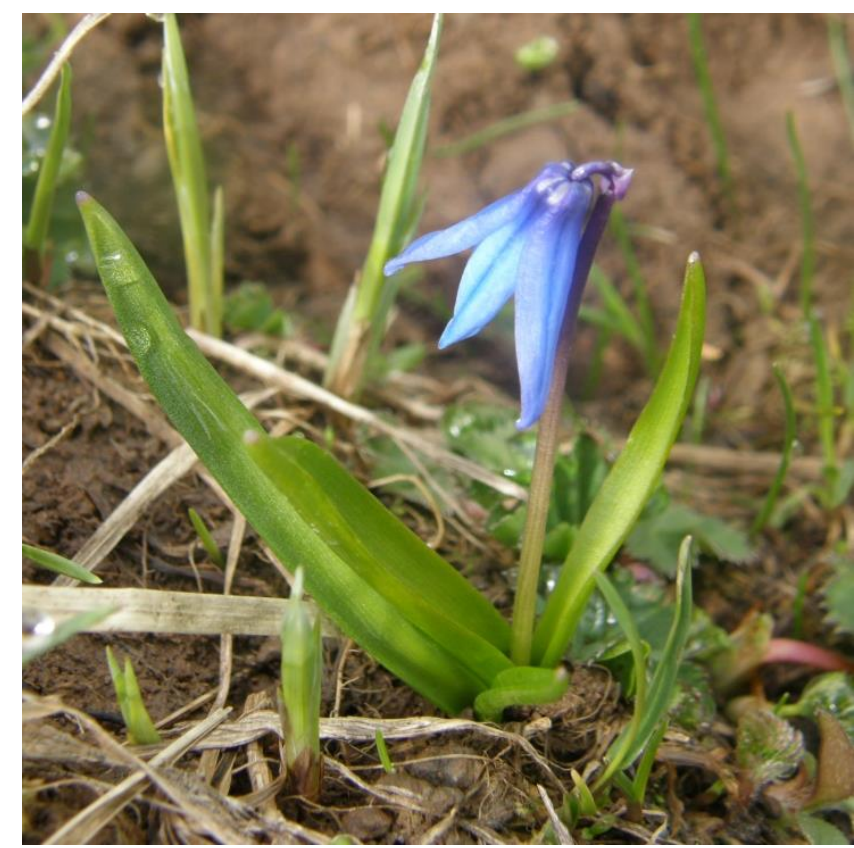

Figure 8. Scilla siberica subsp. armena, $2267 \mathrm{~m}$

\section{CONCLUSION}

Geophytes are a group of plants, which remain underground for the majority of the year, brings continuity to the gardens with beautiful and glamorous flowers during spring and autumn. With their use in flowing masses, in groups and in sets, the geophytes are useful for the creation of wide flower beds, the filling of the gaps between shrubs and bush groups, and the creation of a natural look for the environment. In landscape design and applications, geophytes can be used in borders, lawn areas, rock and stone gardens, flower beds, scent gardens, and interiors. In addition to focusing on the use in landscape applications of the geophytes, which present a natural distribution and have a rich diversity in Turkey; adoption 
and cultivation efforts should be developed in order to ensure and protect the continuity of the species, and to introduce new species (Seyidoğlu et al. 2009).

According to this study, the geophytes which can be used as ornamental plant have potential for economy. Further studies should be carried out on the geophytes of the province, especially to explore for cultivation, so that they can contribute to the economy significantly. They have been under many risks of land clearing, overgrazing and agricultural pests. So, different conservation approaches such as educational activities to increase public awareness should be implemented in the province for sustainable development.

\section{Acknowledgements}

This research was partially supported by the Research Fund of Istanbul University (Project No. 1441) and partially conducted at author's own expense.

\section{References}

Altundağ, E. (2010). Iğdır'in Faydalı ve Zehirli Bitkileri. Malatya, Medipres Yayıncılık.

Avcu, C., Selvi, S., Satıl, F. (2016). Katran Dağ1 (Bayramiç/Çanakkale) ve Çevresinde Yayılış Gösteren Geofit Bitkiler ve Ekolojik Özellikleri. Iğdır Üni. Fen Bilimleri Ens. Der., 6(3), 9-16.

Çelik, A., Çiçek, M., Semiz, G., Karıcalı, M. (2004). Taxonomic and Ecological Investigation on Some Geophytes Growing Around Denizli Province (Turkey). Turk J Bot, 28, 205-211.

Çığ, A., Başdoğan, G. (2015). In vitro propagation Techniques for some geophyte ornamental plants with high economic value. International Journal of Secondary Metabolite. 2(1), 27-49.

Çıngay, B., Ataşlar, E., Koyuncu, O. (2012). Geophytes of Yazılıkaya (Han-Eskişehir, Turkey). Bocconea, 24, 227-230.

Davis, P.H. (1965-1985). Flora of Turkey and the East Aegean Islands. Vol. 1-9, Edinburgh, University Press.

Davis, P.H., Mill, R.R., Tan, K. (1988). Flora of Turkey and the East Aegean Islands. Vol. 10 (Suplement I), Edinburgh, University Press.

Demirelma H., Ertuğrul, K. (2016). The geophytes of the region between Derebucak (Konya/Turkey)Ibradi and Cevizli (Antalya/Turkey). BioDiCon, 9(3), 52-57.

Eker, I., Koyuncu, M., Akan, H. (2008). The Geophytic Flora of Şanliurfa Province, Turkye. Turk J Bot, 31, 367-380.

Ekim, T., Koyuncu M., Güner, A., Erik, S., Yıldız, B., Vural M. (1991). Türkiye'nin Ekonomik Değer Taşıyan Geofitleri Üzerinde Taksonomik ve Ekolojik Araştırmalar. Ankara.

Ekim, T., Koyuncu, M., Vural, M., Duman, H., Aytaç, Z., Adigüzel, N. (2000). Türkiye'nin Bitkileri Kırmız1 Kitabı (Red Data Book of Turkish Plants). Ankara.

Fırat, M., Karavelioğulları, F.A., Aziret, A. (2015). Geophytes of East Anatolia (Turkey). MJAL 5(1), 38-53.

Güner, A., Özhatay, N., Ekim, T., Başer, K.H.C. (2000). Flora of Turkey and the East Aegean Islands.Vol 11, Edinburgh, University Press.
Kamenetsky, R., Hiroshi, O. (2013). Introduction. In: Kamenetsky, R., Hiroshi O. (Eds), Ornamental Geophytes From Basic Science to Sustainable Production., CRC Press, U.S., pp. 15-19.

Korkmaz, M., Alpaslan, Z., Turgur, N., İlhan, V. (2014). Ethnobotanical Aspects of Some Geophytes From Ergan Mountain, Turkey. Bangladesh J. Bot, 43(3), 315-321.

Özhatay, N., Kültür, Ş., Gürdal, B. (2013). Check-list of additional taxa to the supplement Flora of Turkey. J Fac Pharm Istanbul 43(1): 33-82.

Özuslu, E., İskender, E. (2009). Geophytes of Sof Mountain (Gaziantep/Turkey). BioDiCon, 2(2), 78-84.

Sargin, A.A., Selvi, S., Akçiçek E. (2013). Alaşehir (Manisa) ve Çevresinde Yetişen Bazı Geofitlerin etnobotanik Açıdan İncelenmesi. Erciyes Üniversitesi Fen Bilimleri Enstitüsü Dergisi, 29(2), 170-178.

Seyidoğlu, N., Zencirkıran, M.,Ayaşlıgil, Y. (2009). Position and application areas of geophytes within landscape design. AJAR, 4(12), 13511357.

The Plant List (Internet), (2013). (cited 2017 March 10), Avaliable from: http://www.theplantlist.org

Turkish Plants Data Service (Internet), (cited 2017 January), Avaliable from: http://www.tubives.com

Submitted: 20.04.2017

Accepted: 01.06.2017 